\title{
Associations of aspirin, statins and metformin with lung cancer risk and related mortality
}

\section{Commentary on: \\ Kang J, et al. The associations of aspirin, statins, and metformin with lung cancer risk and related mortality: a time-dependent analysis of population-based nationally representative data. J Thorac Oncol 2021; 16: 76-88.}

\section{Context}

Cardiovascular diseases (CVDs), including ischaemic heart disease and cerebrovascular disease, are the leading cause of death in both sexes worldwide, followed by cancer [1, 2]. Most CVDs can be prevented by addressing behavioural risk factors, such as tobacco smoking, unhealthy diets, obesity, physical inactivity and harmful use of alcohol [3]. Individuals with CVDs or those at high cardiovascular risk due to the presence of one or more risk factors (e.g. arterial hypertension, diabetes, hyperlipidaemia and obesity) require early detection and management [3]. Counselling and medicines should be offered, as appropriate. These shared metabolic and behavioural risk factors are causatively linked to the development of heart disease, stroke, cancer, diabetes and respiratory diseases [3]. Lung cancer is, by far, the most common cause of cancer-related death worldwide [3, 4]. Given that lung cancer patients may initially present with cardiovascular and/ or metabolic comorbidities at the time of cancer diagnosis, questions have been raised as to whether

@ERSpublications

Given the poor survival of lung cancer patients and the promising observations herein, future studies (RCTs) should further investigate both time- and dose-dependent effects of combination therapies across all categories of prevention of lung cancer. https://bit.ly/3hlYTtY long-term use of combined medications to treat these diseases affects the incidence and mortality of lung cancer patients on a population-based level.

Established oral therapies are currently the cornerstone of prevention of cardiovascular and metabolic diseases, and include: 1) the antithrombotic agent acetylsalicylic acid (also known as aspirin) for reducing CVD morbidity and mortality among survivors of myocardial infarction and stroke; 2) the glucose-lowering agent metformin as the first-line therapeutic agent for type 2 diabetes (T2D); and 3) the lipid-lowering statins as effective drugs for reducing the risk of myocardial infarction, ischaemic stroke, and development of peripheral arterial disease.

The impact of these medications on the present management of cardiovascular and metabolic diseases is emphasised by the fact that these agents are in the World Health Organization model list of essential medicines [5]

Over the past few decades, these groups of drugs have commonly been prescribed together as a measure of chemoprevention given the close interconnection between cardiovascular disease, diabetes and dyslipidaemia [3, 6]. Nevertheless, in the literature to date, both observational and randomised controlled trials (RCTs) have merely assessed the individual potential effects of each of these three types of agents, without taking into account their combined effect, dose-response relationship or time-dependent exposure [7-10]. The study to be discussed here by KANG et al. [11] has shed some light on the latter.
Cite as: Frille $A$, Costantini $A$ Sreter KB. Associations of aspirin, statins and metformin with lung cancer risk and related mortality. Breathe 2021; 17: 200325.

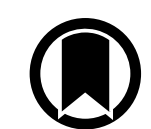

CrossMark 


\section{Methods}

In this nationwide, population-based, retrospective observational cohort study, the investigators employed the database of the Korean National Health Insurance Services (NHIS). It included 732199 Koreans who participated in a large national health screening programme between the years 2002 and 2003 . The study participants were pooled from three NHIS cohort datasets: NHIS Senior, NHIS Health Screening Cohort and NHIS National Sample Cohort. Patients who died of any cause or had any cancer diagnosis before 1 January 2004 , or were aged $<40$ or $\geq 80$ years in 2002 to 2003 were excluded from the study.

The primary outcome measures of the study were lung cancer incidence and mortality. Lung cancer incidence was identified using a registered lung cancer diagnosis code (i.e. (34) from the 10th revision of the International Classification of Diseases (ICD). Lung cancer mortality was determined using the Korean National Death Registry. The study subjects were followed from 1 January 2004 (index date) to the date of lung cancer diagnosis (incidence) and death (mortality), or death from any other cause, or until 31 December 2013. The cumulative duration of use of cardiovascular (i.e. aspirin and statins) and anti-diabetic medications (i.e. metformin) was analysed with a 2-year latent period. The timedependent exposure to these three medications was defined as follows: 1) the cumulative duration of drug use equals the total number of days of drug exposure; and 2) the cumulative defined daily dose (cDDD). The cumulative duration of drug use and cDDD were calculated for each 2-year period and participants were categorised based on the duration of medication exposure (i.e. nonusers, <0.5-1 year, $1-1.5$ years, $1.5-2$ years, $\geq 2$ years or $c D D D)$.

Medication exposure was considered a timedependent variable in a Cox proportional hazards model. The individual effect of the cumulative dose of aspirin, statins, or metformin on the incidence and mortality of lung cancer was assessed by considering concomitant use of other cardiovascular drugs. To determine the independent associations of each of the three medications with lung cancer risk, two models were used to adjust for the covariates of age, sex, income, body mass index, smoking status, alcohol use and severity of comorbidity (Charlson comorbidity index), as well as for other medication use. In addition, stratified analyses by smoking status were performed to test the associations between aspirin, statins and metformin use, and lung cancer risk and mortality.

This was the first published study to evaluate the combined impact of aspirin, statins, and metformin on lung cancer incidence and mortality. To address these combined associations, the authors categorised the cohort into eight groups based on medication exposure, accounting for the possible concomitant use of these three drugs.

\section{Main results}

After the 10-year follow-up period, a total of 5990 lung cancer cases and 5938 deaths from lung cancer over the follow-up years was observed. Of the 732199 participants, 9.0\% ( $n=66024)$, $5.1 \%(n=37031)$ and $6.3 \%(n=46205)$ were ever users of aspirin, statins or metformin, respectively. Moreover, $6.3 \%(n=46205)$ of all participants were diagnosed with T2D, of which $55.8 \%$ were ever on metformin.

There was no significant association between ever use of aspirin and incidence of lung cancer or lung cancer-related mortality. Nevertheless, aspirin showed a risk reduction for deaths of lung cancer patients when taken for $\geq 1.5$ years.

Lung cancer incidence was not associated with ever use or CDDD use of statins. However, a dosedependent reduction in lung cancer-related mortality was observed with increased cDDD of statin use; a cumulative use of statins for $\geq 1.5$ years led to a relative risk reduction of $23 \%$. Ever use of metformin was significantly associated with reduced incidence of lung cancer compared with non-diabetic patients (adjusted hazard ratio (aHR) 0.89). Diabetic patients with a cumulative metformin use of $\geq 1.5$ years showed both a decreased lung cancer incidence (aHR 0.44) and mortality (aHR 0.76) compared to diabetic patients who did not take metformin. In a dose-dependent manner among diabetic patients, metformin use had a protective association with lung cancer incidence and mortality. The association between metformin and decreased lung cancer mortality was strongest in nonsmokers (aHR 0.41 in the group with $\geq 1.5$ years of $C D D D$ ) and women (aHR 0.19 in the group with $\geq 1.5$ years of cDDD) compared with the entire cohort.

Concomitant use of aspirin, statins and metformin appeared to protect against the development of lung cancer or lung cancer death. Ever users of this combination regimen were associated with decreased lung cancer incidence (aHR 0.83) and mortality (aHR 0.83) compared to never users. The magnitude of the effect increased progressively with a longer duration of combined medication exposure. Those subjects with $\geq 1.5$ years of combination drug use showed the lowest risk for developing lung cancer (aHR 0.49 ) and associated mortality (aHR 0.42). Smoking status did not influence these inverse associations. Table 1 summarises the main results.

\section{Commentary}

Prevention of lung cancer is a priori the pivotal measure to reduce the numerous morbidities and enormous mortality in patients with lung cancer, as well as its burden on individual patients, their families and society. The most effective preventive measure is tobacco cessation since the causal chain for tobacco smoking leading to lung cancer is well 
Table 1 Summary of results

\begin{tabular}{|c|c|c|}
\hline Medication & Incidence & Mortality \\
\hline \multirow[t]{2}{*}{ Aspirin } & No effect & No effect \\
\hline & & $\begin{array}{l}\text { Risk reduction of } 13-19 \% \text { for deaths of lung cancer patients } \\
\text { when taken } \geq 1.5 \text { years ( } a \mathrm{HR}^{\#} 0.81 \text { and aHR } 0.87 \text { ) }\end{array}$ \\
\hline Statins & No effect & $\begin{array}{l}\text { Dose-dependent risk reduction of } 23 \% \text { in subjects with } \\
\text { cumulative use for } \geq 1.5 \text { years (aHR } \# \text {, } 0.77)\end{array}$ \\
\hline \multirow[t]{2}{*}{ Metformin } & Reduced for ever-users (aHR 0.89 ) & $\begin{array}{l}\text { Reduced risk in diabetic subjects with } \geq 1.5 \text { years metformin use } \\
(\mathrm{aHR} \| \text { 0.76) compared to those who did not take metformin }\end{array}$ \\
\hline & $\begin{array}{l}\text { Reduced in subjects with } \geq 1.5 \text { years of } \\
\text { metformin use (aHR } 0.44)\end{array}$ & \\
\hline \multirow[t]{2}{*}{ Triple combination } & Reduced for ever-users (aHR\# 0.83) & Reduced for ever-users (aHR $\mathrm{R}^{\#}$ 0.83) \\
\hline & $\begin{array}{l}\text { Reduced in subjects with } \geq 1.5 \text { years of } \\
\text { combination drug use }\left(\mathrm{aHR}^{\#} 0.49\right)\end{array}$ & 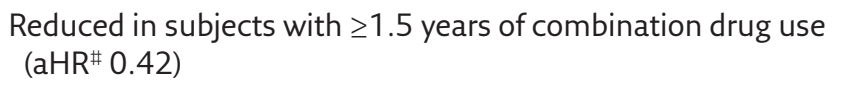 \\
\hline
\end{tabular}

aHR: adjusted hazard ratio. \#: adjustment for age, sex, income, body mass index, smoking, alcohol comsumption and Charlson comorbidity index; 9 : additional adjustment for other medication use (e.g. aspirin use was adjusted for statin use and metformin use).

established, accounting for about $80-90 \%$ of all lung cancer cases [12].

A further preventive strategy is chemoprevention, i.e. the use of dietary or pharmaceutical interventions to slow or reverse the progression of premalignancy to invasive cancer [13, 14]. Preventive measures apply to common diseases and are generally aimed at: 1) primary prevention: subjects who are at high risk (current or former smokers), but without evidence of a disease, e.g. lung cancer or CVD; 2) secondary prevention: subjects who have risk factors and show evidence of early stage disease, e.g. premalignancy; and 3) tertiary prevention: subjects with pre-existing, risk factor-related diseases (e.g. lung cancer, CVD) to prevent secondary events (e.g. second primary tumour, myocardial infarction, stroke).

The rationale for chemoprevention is based on two main concepts: 1) multistep carcinogenesis (a series of genetic and epigenetic alterations leading to malignant transformation from normal cells to precursor cells and then to invasive tumour cells); and 2) field cancerisation (assuming that many areas of the aerodigestive tract are simultaneously at risk of cancer formation due to carcinogen exposure) $[14,15]$.

As reviewed by the authors, long-term follow-up data from some trials of these drugs in the treatment and prevention of CVD, diabetes or dyslipidaemia have provided an opportunity to explore their possible benefits on cancer incidence and mortality. Unfortunately, Cochrane-initiated systematic reviews and meta-analyses investigating the anti-tumour effects of these agents alone or in combination in patients with lung cancer are still not yet available. The latest analysis on the chemoprevention of lung cancer was published within the American College of Chest Physicians evidence-based clinical practice guidelines in 2013. It concluded that none of the phase 3 trials with these agents, including aspirin or metformin, provided sufficient evidence to be used in the chemoprevention of lung cancer so far [14].

\section{Aspirin}

The effects of aspirin on the inhibition of the cyclooxygenases (COX) 1 and 2 are dose-dependent. At lower doses, aspirin inhibits COX-1, leading to reduced production of thromboxane A2 and, thus, producing a clinically relevant anti-platelet effect. Higher doses also inhibit COX-2 that blocks prostaglandin production, leading to analgesic and antipyretic effects. Plausible biological mechanisms for anti-tumour effects of aspirin include induction of cell apoptosis and inhibition of COX-catalysed production of prostaglandin, which in turn is associated with tumour cell angiogenesis, cell proliferation and inhibition of immune surveillance [16]. Credible evidence exists that aspirin possibly reduces the risk of colorectal cancer over long-term follow-up with $>10$ years of treatment [17-19]. However, in this study, aspirin only reduced lung cancer incidence when prescribed in combination with metformin and a statin. In addition, aspirin showed a risk reduction for deaths of lung cancer patients when taken for $\geq 1.5$ years or in combination with metformin and a statin. Moreover, the risks for lung cancer incidence or mortality appeared to decrease over time when prescribed as a three-fold combination therapy.

\section{Metformin}

Belonging to the guanidine derivatives, metformin's pharmacodynamic profile in the treatment of T2D is truly versatile, ranging from decreasing hepatic glucose output by inhibiting gluconeogenesis and increasing insulin-mediated glucose utilisation in peripheral tissue (e.g. muscle and liver) to having 
an anti-lipolytic effect that lowers serum fatty acid concentrations as well as an anti-thrombotic effect [20]. All these effects lead to the reduced progression of impaired glucose tolerance to T2D, reduced fasting hyperinsulinaemia, reduced myocardial infarction, increased survival in T2D patients, reduced insulin resistance, and modestly improved blood lipid profile [21].

Among the other metabolic effects, on a molecular basis, metformin activates the enzyme adenosine monophosphate-activated protein kinase (AMPK), leading to increased expression and phosphorylation of p53, thus resulting in cell cycle arrest and/or apoptosis. Therefore, activation of AMPK is one possible mechanism by which metformin exerts its potential anti-neoplastic properties [22].

In the present study, patients with diabetes who had taken metformin for $>1.5$ years were observed to have a relative risk reduction of $56 \%$ in the incidence and $24 \%$ in the mortality of lung cancer compared to participants without diabetes.

\section{Statins}

Statins, such as simvastatin or atorvastatin, are competitive inhibitors of hydroxymethylglutaryl coenzyme $A$ reductase, the rate-limiting step in the intrahepatic cholesterol synthesis as part of the mevalonate pathway. Besides cholesterol, this pathway produces the protein anchor molecules isoprenoids, such as farnesyl pyrophosphate. Among others, they bind to Ras proteins, facilitating protein translocation as well as protein-protein and protein-membrane interactions [23]. Statins deplete the cellular pools of isoprene precursors and reduce the isoprenylation of Ras proteins. By inhibiting the proper function of Ras proteins and others, statins exert growth inhibitory, proapoptotic, anti-invasive, anti-angiogenic and pro-differentiation responses in neoplastic cells of diverse origins [23].

Even though observational studies suggest a decreased risk of mortality and improved overall survival in lung cancer patients, no convincing evidence from meta-analyses of RCTs exists to date to show that statins increase or decrease the risk of cancer, or lung cancer in particular [24].

In the present study, statin administration was not associated with reduced lung cancer incidence in a dose-independent manner. However, mortality was reduced in both a dose- and time-dependent fashion. Unfortunately, the authors did not provide information about which statin was administered. Analyses conducted on a wide range of tumour cell lines suggest that lipophilic statins, such as simvastatin, show pronounced anti-tumour effects, whereas hydrophilic statins, such as pravastatin, do not have cytotoxic effects on these tumour cell lines [23]. Nonetheless, the authors of the present study recognise that the register database did not allow for information on statin derivatives.

\section{Combined medication over a period of time}

A key factor in study trial design is the duration of treatment and follow-up required to detect significant differences in the incidence of cancer between treatment groups. Long trial times and a large number of participants needed to achieve adequate power means that chemoprevention trials are expensive and laborious [17]. The authors of the present study investigated the combined use of three groups of drugs with anti-tumour potential in a Korean population of more than 700000 participants over a lengthy period of 10 years. Participants medicated with the triple combination of aspirin, metformin and statin showed both a dose- and time-dependent relative risk reduction for lung cancer incidence and mortality of $51 \%$ and $58 \%$, respectively. Previous meta-analyses of RCTs conducted on various tumour entities have led to the conclusion that the duration and dose of medications must be adequately appreciated in order to estimate the effect of these agents more accurately [18, 25-28].

As the authors herein hypothesised, the concomitant use of the triple therapy resulted in favourable effects on lung cancer risk and mortality by the concurrent inhibition of multiple pathways related to lung cancer cell growth and proliferation, potentially leading to synergistic anti-tumour effects.

\section{Pending points}

Some limitations of the study should be underlined. Any study that relies on data derived from administrative claims is prone to be biased by confounding factors, especially with regards to lung cancer diagnosis as was the case here. The diagnosis in this study was considered only if the patient received treatment for lung cancer. As such, patients unfit for lung cancer treatment would not have been included in the analysis. This could have led to the non-inclusion of patients with a presumably heavy history of cardiovascular disease or diabetes. Although this bias is possible, it may also have led to the most treatment-fit patients being analysed and thus giving a good glimpse of actual clinical practice. Another bias due to the administrative nature of the study would be that information on lung cancer histology and molecular alterations at diagnosis were not provided. Such data would have given a deeper insight into the potential signalling pathways used by these agents. For aspirin and metformin, the results from at least two RCTs and one cohort study suggest that patients with adenocarcinoma benefit from chemoprevention in terms of improved survival $[18,29,30]$.

Moreover, the authors themselves acknowledge that the lung cancer incidence was likely underestimated due to the reliance on a single ICD code and reimbursement data to identify potential subjects. Given that the data were collected for medical service claims and reimbursement, the 
authors caution readers that the information on diagnosis and disease might not be optimal for sufficiently identifying disease occurrence and prevalence [31]. These and other limitations were discussed by the authors in their previous papers detailing the cohort design and national databases used in their current research [31, 32].

The design of the study has a number of unclear aspects that may help explain some of the main findings. First, it is difficult to assess whether there was complete follow-up for all subjects in the study with no opportunity for some to be lost to follow-up. Censoring for this may impact the interpretation of the key outcomes of the study. Secondly, respiratory conditions were not identified in the study. It is possible that these patients may be more likely to have lung cancer detected than those without any respiratory complaints. Thirdly, as mentioned by the authors in their previous study, the disease diagnosis variables in the healthcare claim data might not accurately reflect the patients' medical conditions [32]. Finally, the study neither identifies the conditions most commonly indicated for the three drugs nor the conditions for which they were prescribed for the patients.

Furthermore, the modality of treatment for the lung cancer was not taken into consideration by the authors. Any surgical intervention or anticancer therapy administered following lung cancer diagnosis could have confounded the relationship between these drugs and cancer-related death.

Moreover, a stratified analysis by cancer stage at the time of lung cancer diagnosis could allow speculation as to whether chemoprevention reduces the proportion of advanced stages at the time of diagnosis.

In addition, studies that rely on registers and databases are not per se designed to explore cancer outcomes, making inference imprecise. Trials of chemoprevention could be improved by better identification of individuals at high risk for tumours.

\section{Implication for practice}

This study brings more data to the mounting evidence that the use of metformin seems to be beneficial with regards to survival in patients with lung cancer. Furthermore, it brings innovative data with regards to aspirin, statins and especially the combination of aspirin, statins, and metformin in terms of lung cancer incidence and mortality in a large population-based cohort.

Although these results seem promising, the direct implications for current clinical practice remain unclear as several questions persist. For instance, due to the nature of the observational study, more evidence is needed before aspirin, statins or metformin alone, or their combinations, can be offered to patients to prevent lung cancer (i.e. chemoprevention). It is also not possible at this time, based on the data in the study, to recommend that aspirin, statins or metformin alone, or in combination, should be offered to patients with lung cancer as a means of tertiary prevention.

Therefore, research should be encouraged in this field by conducting RCTs to determine whether these drugs really do have a preventive effect and can offer mortality reduction. These trials would need to focus on which drug alone or in a drugdrug association effectively reduces lung cancer incidence and mortality. Furthermore, the duration of the chosen treatments should also be explored as well as the dose and the date of introduction of the medication(s) with respect to the date of cancer diagnosis. These studies would most likely be challenging to perform in terms of time and related costs, but could provide crucial answers related to the field of lung cancer prevention and treatment. Perhaps future lung cancer guidelines will be able to address the questions of chemoprevention by means of high quality observational and treatment studies.

\section{Affiliations}

\section{Armin Frille ${ }^{1}$, Adrien Costantini ${ }^{2}$, Katherina B. Sreter ${ }^{3}$}

${ }^{1}$ Dept of Respiratory Medicine, University of Leipzig, Leipzig, Germany. ${ }^{2}$ Dept of Respiratory Diseases and Thoracic Oncology, APHP-Hôpital Ambroise Paré, Boulogne-Billancourt, France. ${ }^{3}$ Dept of Clinical Immunology, Pulmonology and Rheumatology, University Hospital Centre "Sestre Milosrdnice", Zagreb, Croatia.

\section{Conflict of interest}

A. Frille has nothing to disclose. A. Costantini has nothing to disclose. K.B. Sreter has nothing to disclose.

\section{Support statement}

A. Frille was supported by the postdoctoral fellowship "MetaRot program" from the Federal Ministry of Education and Research (BMBF), Germany (FKZ 01E01501, IFB AdiposityDiseases), a research grant from the Mitteldeutsche Gesellschaft für Pneumologie (MDGP) e.V. (2018-MDGP-PA-002), and a junior research grant from the Medical Faculty, University of Leipzig. He was a scholar of the German Society of Internal Medicine (DGIM, Roland-Müller academy). 


\section{References}

1. World Health Organization. The global burden of disease: 2004 update. Geneva, World Health Organization, 2008.

2. World Health Organization. Cardiovascular diseases (CVD). www.who.int/en/news-room/fact-sheets/detail/ cardiovascular-diseases-(cvds) Date last updated: 17 May 2017; date last accessed: 5 December 2020.

3. World Health Organization. Global Atlas on Cardiovascular Disease Prevention and Control. Geneva, World Health Organization, 2011.

4. Bray F, Ferlay J, Soerjomataram I, et al. Global cancer statistics 2018: GLOBOCAN estimates of incidence and mortality worldwide for 36 cancers in 185 countries. CA Cancer J Clin 2018; 68: 394-424.

5. World Health Organization. Model List of Essential Medicines, 21st List, 2019. Geneva, World Health Organization, 2019.

6. Grant PJ, Cosentino F. The 2019 ESC Guidelines on diabetes, pre-diabetes, and cardiovascular diseases developed in collaboration with the EASD: new features and the "Ten Commandments" of the 2019 Guidelines are discussed by Professor Peter J. Grant and Professor Francesco Cosentino, the Task Force chairmen. Eur Heart J 2019; 40: 3215-3217.

7. Ratnasinghe LD, Graubard BI, Kahle L, et al. Aspirin use and mortality from cancer in a prospective cohort study. Anticancer Res 2004; 24: 3177-3184

8. Yang TY, Lin WM, Lin CL, et al. Correlation between use of simvastatin and lovastatin and female lung cancer risk: a nationwide case-control study. Int J Clin Pract 2015; 69 : 571-576.

9. Nielsen SF, Nordestgaard BG, Bojesen SE. Statin use and reduced cancer-related mortality. N Engl J Med 2013; 368 : 576-577.

10. Yao L, Liu M, Huang Y, et al. Metformin use and lung cancer risk in diabetic patients: a systematic review and metaanalysis. Dis Markers 2019; 2019: 6230162.

11. Kang J, Jeong S-M, Shin DW, et al. The associations of aspirin, statins, and metformin with lung cancer risk and related mortality: a time-dependent analysis of populationbased nationally representative data. J Thorac Oncol 2021; 16 76-88.

12. Alberg AJ, Brock MV, Ford JG, et al. Epidemiology of lung cancer: diagnosis and management of lung cancer, 3rd ed: American College of Chest Physicians evidence-based clinical practice guidelines. Chest 2013; 143: e1S-e29S.

13. Sporn MB, Dunlop NM, Newton DL, et al. Prevention of chemical carcinogenesis by vitamin $\mathrm{A}$ and its synthetic analogs (retinoids). Fed Proc 1976; 35: 1332-1338.

14. Szabo E, Mao JT, Lam S, et al. Chemoprevention of lung cancer: diagnosis and management of lung cancer, 3rd ed: American College of Chest Physicians evidence-based clinical practice guidelines. Chest 2013; 143: e40S-e60S.

15. Slaughter DP, Southwick HW, Smejkal W. Field cancerization in oral stratified squamous epithelium; clinical implications of multicentric origin. Cancer 1953; 6: 963-968.

16. Dannenberg AJ, Lippman SM, Mann JR, et al Cyclooxygenase-2 and epidermal growth factor receptor: pharmacologic targets for chemoprevention. / Clin Oncol 2005; 23: 254-266

17. Cuzick J, Otto F, Baron JA, et al. Aspirin and non-steroidal antiinflammatory drugs for cancer prevention: an international consensus statement. Lancet Oncol 2009; 10: 501-507.

18. Rothwell PM, Fowkes FG, Belch JF, et al. Effect of daily aspirin on long-term risk of death due to cancer: analysis of individual patient data from randomised trials. Lancet 2011 ; 377: 31-41.

19. Algra AM, Rothwell PM. Effects of regular aspirin on long-term cancer incidence and metastasis: a systematic comparison of evidence from observational studies versus randomised trials. Lancet Oncol 2012; 13: 518-527.

20. Rena G, Hardie DG, Pearson ER. The mechanisms of action of metformin. Diabetologia 2017; 60: 1577-1585.

21. Bailey CJ. Metformin: historical overview. Diabetologia 2017; 60: 1566-1576

22. Aljada A, Mousa SA. Metformin and neoplasia: implications and indications. Pharmacol Ther 2012; 133: 108-115.

23. Osmak M. Statins and cancer: current and future prospects. Cancer Lett 2012; 324: 1-12.

24. Xia D-K, Hu Z-G, Tian Y-F, et al. Statin use and prognosis of lung cancer: a systematic review and meta-analysis of observational studies and randomized controlled trials. Drug Des Devel Ther 2019; 13: 405-422.

25. Xu J, Yin Z, Gao W, et al. Meta-analysis on the association between nonsteroidal anti-inflammatory drug use and lung cancer risk. Clin Lung Cancer 2012; 13: 44-51.

26. Hochmuth F, Jochem M, Schlattmann P. Meta-analysis of aspirin use and risk of lung cancer shows notable results. Eur J Cancer Prev 2016; 25: 259-268.

27. Zhang Z-J, Bi Y, Li S, et al. Reduced risk of lung cancer with metformin therapy in diabetic patients: a systematic review and meta-analysis. Am J Epidemiol 2014; 180: 11-14.

28. Gandini S, Puntoni M, Heckman-Stoddard BM, et al. Metformin and cancer risk and mortality: a systematic review and meta-analysis taking into account biases and confounders. Cancer Prev Res (Phila) 2014; 7: 867-885.

29. Slatore CG, Au DH, Littman AJ, et al. Association of nonsteroidal anti-inflammatory drugs with lung cancer: results from a large cohort study. Cancer Epidemiol Biomarkers Prevent 2009; 18: 1203-1207.

30. Arrieta O, Barron F, Padilla M-AS, et al. Effect of metformin plus tyrosine kinase inhibitors compared with tyrosine kinase inhibitors alone in patients with epidermal growth factor receptor-mutated lung adenocarcinoma: a phase 2 randomized clinical trial. JAMA Oncol 2019; 5: e192553.

31. Cheol Seong S, Kim Y-Y, Khang $\mathrm{Y}-\mathrm{H}$, et al. Data resource profile: the National Health Information Database of the National Health Insurance Service in South Korea. Int Epidemiol 2017; 46: 799-800.

32. Seong SC, Kim YY, Park SK, et al. Cohort profile: the National Health Insurance Service-National Health Screening Cohort (NHIS-HEALS) in Korea. BMJ Open 2017; 7: e016640. 